\title{
Aeroservoelastic Optimization under Stochastic Gust Constraints
}

\author{
Bret K. Stanford* \\ NASA Langley Research Center, Hampton, VA, 23681
}

\begin{abstract}
This work considers the aeroservoelastic optimization of a highly flexible transport aircraft wingbox with several control surfaces distributed along the trailing edge. The steady deflections of the control surfaces are designed to alleviate static maneuver loads, while the unsteady deflections are designed to alleviate stochastic continuous gust disturbances. Spatially-detailed unsteady stochastic stress and panel buckling constraints are formulated via modal acceleration, and by methods to locate the most-probable failure point along an equal-probability hypersurface. For the case considered here, it is found that the inclusion of such gust constraints during optimization presents a sizable structural mass penalty. In some cases, this mass penalty can be completely recovered with controlled gust load alleviation.
\end{abstract}

\section{Introduction}

Modern aeroservoelastic optimization typically considers the simultaneous design of multiple subsystems, including the wing jig shape details, the structural wingbox sizing, control surface scheduling, and control law architecture: see for example Refs. 1 - 5, and a broader summary in Ref. 6. In these papers, articulated control surfaces are used for active cruise drag reduction, maneuver load alleviation (MLA), gust load alleviation (GLA), and active flutter suppression (AFS), among other vehicle-level flight control system (FCS) uses. The way in which this control system should be optimized is tightly coupled to the design decisions made for the structural sizing, jig shape details, etc.

One focus of this work is on the design interplay between the different load cases, particularly between maneuver loads and gust loads. It should be expected that the inclusion of MLA in the design process will allow an optimizer to feasibly reduce structural weight, since the loads borne by the wingbox will be reduced. This in turn will amplify the role of flutter and gust constraints during the optimization (i.e., make the constraints harder to satisfy), which can in turn be attenuated through the use of AFS and/or GLA. A second focus of this work is the implementation of stochastic (continuous) stress- and buckling-based gust constraints for spatially-detailed structural wingbox design, as well as control law design, for a generic transport configuration.

It is well known ${ }^{6,7}$ that continuous gust constraints are efficiently developed using state-space methods driven by a white noise input sent through a forming filter. A Lyapunov equation is then solved for the covariance matrix of each aeroservoelastic state. If the design metrics of interest are linear functions of the state vector (such as structural deflections, accelerations, bending moments, etc.), then the covariances and root-mean-squared values of these metrics are rapidly computed for constraint formulation, as done in Refs. 8, 9 , and 10, to name a few. The analytical sensitivities of these terms, typically required for gradient-based optimization, may also be computed. ${ }^{5,7,11}$

For a large-scale structurally-detailed wingbox with complex failure mechanisms, however, accelerations and bending moments may be too simplistic to serve as design constraints. Detailed stress constraints, typically the superposition of $1 g$ cruise stresses and the stochastic gust stresses, introduce two numerical challenges. First, the aeroservoelastic state-space equations must be formulated in a reduced modal space, in order for the computational cost of the Lyapunov solver to remain tractable. Stresses (and their sensitivities) are known ${ }^{12,13}$ to be very difficult to accurately extract from modal approximations, as they converge slowly with mode number. Secondly, stress-based (or buckling-based) failure indices of interest, such as the von

*Research Aerospace Engineer, Aeroelasticity Branch, bret.k.stanford@nasa.gov, AIAA Senior Member. 
Mises stress, are nonlinear quadratic functions of the aerservoelastic state, complicating the formulation of a stochastic failure criterion from the covariance matrix. ${ }^{14,15}$

These two complications have been addressed and solved in Refs 16 and 17, respectively, but neither has been entirely proven out in the context of a complete and realistic aeroservoelastic design exercise, which is the goal of the current work. This will be done for the Common Research Model, a highly flexible transport configuration with several electrohydraulic control surfaces distributed along the trailing edge. Aeroservoelastic design variables include detailed structural sizing variables along the wingbox, steady control surface deflections during MLA, and control law design variables during GLA. The aeroelastic designs are obviously required to be free of flutter, but AFS is not included in this work.

\section{Transport Configuration Test Case}

All of the work in this paper is conducted on a conceptual high aspect ratio Common Research Model (CRM) jig shape shown in Fig. 1. This configuration, described extensively in Ref. 18, has an aspect ratio of 13.5 , a wing span of $72 \mathrm{~m}$, a mean aerodynamic chord of $6.3 \mathrm{~m}$, a taper ratio of 0.25 , a sweep angle of $35^{\circ}$, and a cruise Mach number of 0.85 . The topology of the wingbox in Fig. 1 consists of 58 ribs, leading and trailing spars, and upper and lower surface skins. All shell members are outfitted with T-shaped stiffeners, where the flange is bonded to the shell members. The stiffeners are not modeled explicitly, but instead smeared into the shell stiffness properties. ${ }^{19}$ An aluminum finite element model of this wingbox is composed of 21,300 triangular finite elements. Non-modeled secondary weight (of fasteners, etc.) is captured by increasing the structural density by a factor of 1.25. Nodes along the wing centerline are given a symmetric boundary condition; nodes along the wing root are given a pinned boundary condition.

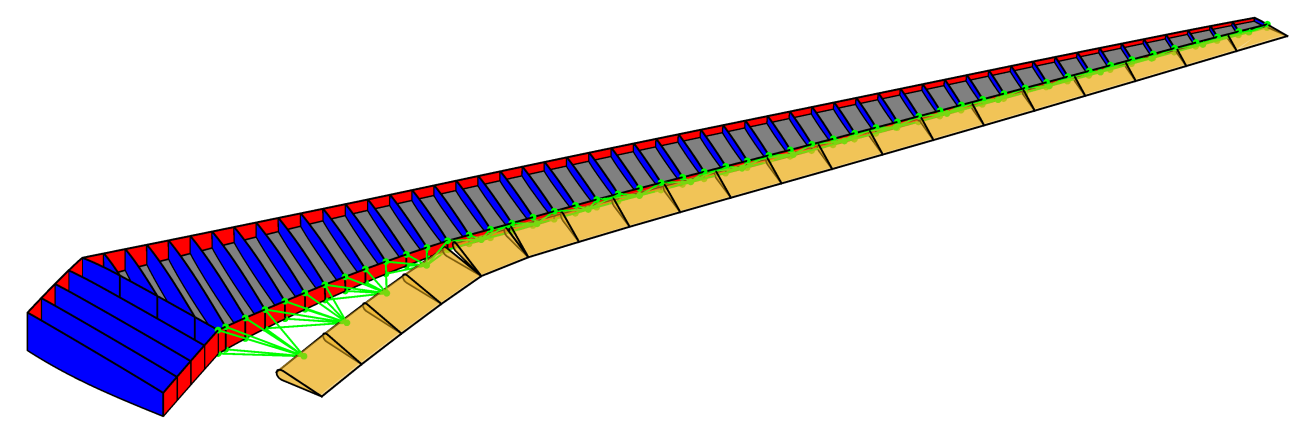

Figure 1. Wingbox and control surfaces of the high aspect ratio CRM.

Twenty articulated control surfaces are distributed along the trailing edge of the wing from root to tip, also seen in Fig. 1. The mass of each electrohydraulic actuator used to drive each control surface is set based on an initial estimate of the hinge moments encountered during maneuver loading, using a constant mass-per-hinge-moment value. This actuator mass value ranges from $214.5 \mathrm{~kg}$ (root) to $26.3 \mathrm{~kg}$ (tip). The mass of each control surface flap is proportional to the surface area of the wedge, and ranges from $140.4 \mathrm{~kg}$ (root) to $35.4 \mathrm{~kg}$ (tip). Actuators are modeled with third-order transfer functions, and are assumed to be irreversible.

The paneling scheme used to model steady and unsteady linear aerodynamic loads over the vehicle is shown in Fig. 2, with a total of 4,150 panels. A finer mesh is used toward the hinge line of the control surfaces, in order to improve the accuracy of the aeroservoelastic loads generated by deflections. Nonstructural lumped mass distributions are also shown in the figure, to model the fuel $(44,800 \mathrm{~kg})$, engine $(11,400 \mathrm{~kg})$, and various unmodeled components in the leading and trailing edges of the wing $(5,480 \mathrm{~kg})$. The total mass of the 20 control surfaces and their actuators is $3,935 \mathrm{~kg}$, and a fixed mass of $63,700 \mathrm{~kg}$ is used to model the remainder of the half-airplane (fuselage, etc.). Typical structural masses (for a single wingbox) will range during optimization from 10,000 to $20,000 \mathrm{~kg}$. The TOGW for the entire vehicle is then roughly $300,000 \mathrm{~kg}$. 


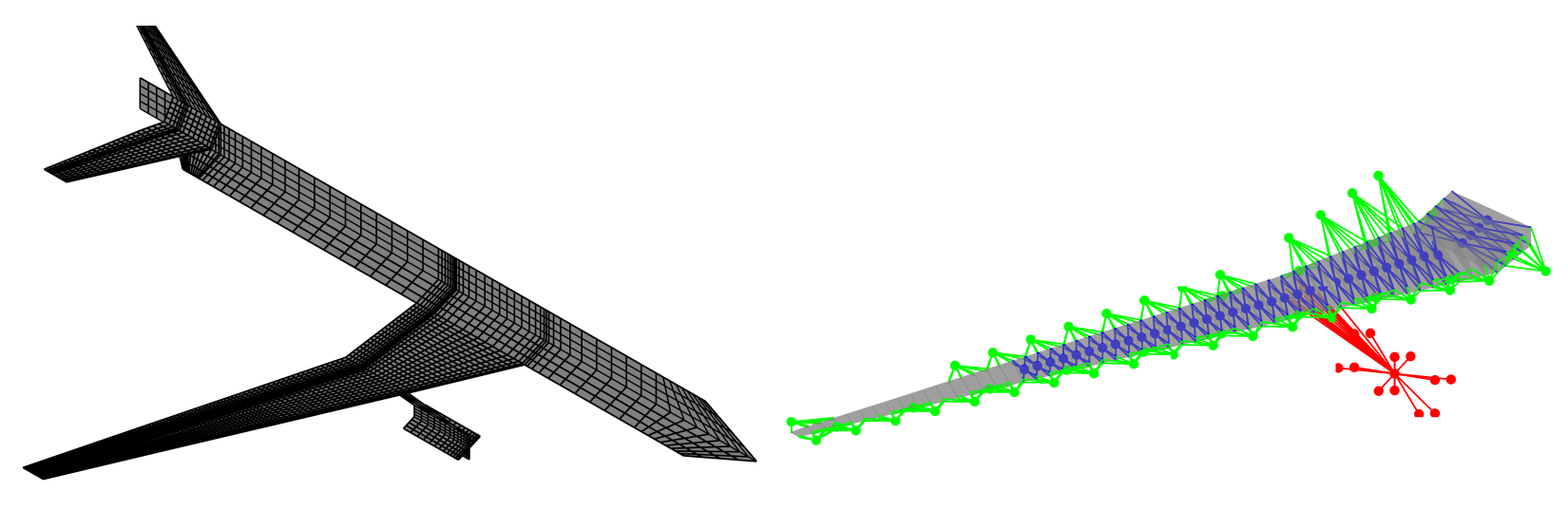

Figure 2. Aerodynamic paneling (left) and non-structural lumped mass distribution (right).

\section{Static Aeroelastic Modeling}

Airplane maneuvers are considered to occur slow enough to warrant only static aeroelastic modeling: dynamic aeroelasticity is ignored in this section. Maneuver loads are computed with the following equation:

$$
\boldsymbol{A}_{s} \cdot \boldsymbol{x}_{s}+\boldsymbol{B}_{s} \cdot \boldsymbol{\theta}=\boldsymbol{F}_{s}
$$

The static aeroelastic solution vector is $\boldsymbol{x}_{s}$, which contains the structural wingbox deformations, $\boldsymbol{u}$, the aerodynamic pressures, $\boldsymbol{C}_{p}$, the trim angle of attack, $\alpha$, and the trim elevator setting $\delta$. The matrix $\boldsymbol{A}_{s}$ contains the finite element stiffness matrix, the aerodynamic influence coefficients, and various load-displacement transfer vectors. The vector $\boldsymbol{\theta}$ is the commanded steady rotation of each trailing edge control surface, and $\boldsymbol{B}_{s}$ transfers the resulting loads onto the aeroelastic system. Finally, $\boldsymbol{F}_{s}$ contains vectors of inertial and thrust forces, jig shape downwash, and trim settings (desired load factor, etc.).

The structural deformation, $\boldsymbol{u}$, is solved in the full-order space, without a modal approximation. Having computed this deformation, plane stresses are computed for each shell finite element:

$$
\left\{\begin{array}{c}
\sigma_{x} \\
\sigma_{y} \\
\sigma_{x y}
\end{array}\right\}_{e}=\boldsymbol{B}_{\sigma_{e}} \cdot \boldsymbol{u}
$$

where the matrix $\boldsymbol{B}_{\sigma_{e}}$ is different for each element $e$. To facilitate a buckling analysis, in-plane tractions may be computed for each stiffened skin panel (delineated by ribs):

$$
\left\{\begin{array}{c}
N_{x} \\
N_{x y}
\end{array}\right\}_{e}=\boldsymbol{B}_{N_{e}} \cdot \boldsymbol{u}
$$

These panel-level tractions are computed via $\boldsymbol{B}_{N_{e}}$, which averages the stress values across the skin panel, and multiplies by the appropriate thickness value. Both global buckling of a stiffened panel (bordered by ribs and spars) and local buckling between each stiffener is accounted for. Quadratic failure surfaces are given by $\boldsymbol{s}^{T} \cdot \boldsymbol{F} \cdot \boldsymbol{s}+\boldsymbol{f} \cdot \boldsymbol{s} \leq 1$. For stresses, the von Mises failure criterion is:

$$
\boldsymbol{F}=\frac{1}{\sigma_{Y}^{2}}\left[\begin{array}{ccc}
1 & -1 / 2 & 0 \\
-1 / 2 & 1 & 0 \\
0 & 0 & 3
\end{array}\right] \quad \boldsymbol{f}=\mathbf{0}
$$

For buckling, these terms are:

$$
\boldsymbol{F}=\frac{1}{N_{x y, c r}^{2}}\left[\begin{array}{ll}
0 & 0 \\
0 & 1
\end{array}\right] \quad \boldsymbol{f}=\frac{1}{N_{x, c r}}\left\{\begin{array}{l}
1 \\
0
\end{array}\right\}
$$


where the critical buckling loads $N_{x, c r}$ and $N_{x y, c r}$ are dependent upon the panel geometry, material properties, thickness, and smeared stiffener properties. Expressions for both local and global critical buckling envelopes are given in Ref. 19.

The von Mises failure index is computed of each of the 21,300 finite elements via Eqs. 2 and 4, and then compressed into a set of 13 Kreisselmeier-Steinhauser (KS) functions: ${ }^{20}$ three for the upper skins, three for the lower skins, three for the ribs, and four for the spars. Similarly, the global and local buckling failure indices are computed for each of the 114 upper and lower skin panels via Eqs. 3 and 5, and then compressed into 8 KS functions: four each for the upper and lower skins. If each of these KS functions is less than one, then the inequality $\boldsymbol{s}^{T} \cdot \boldsymbol{F} \cdot \boldsymbol{s}+\boldsymbol{f} \cdot \boldsymbol{s} \leq 1$ holds.

Six load cases are utilized here, summarized in Table 1 . Load cases 1 and 2 are both run at $2.5 g$, but case 1 features maneuver load alleviation from the control surfaces ( $\boldsymbol{\theta}$ in Eq. 1), whereas case 2 does not: $\boldsymbol{\theta}$ is set to 0 . Forcing the optimizer to account for load cases that do not benefit from control surface-based aeroelastic tailoring (albeit at a lower safety factor) prevents overly-large mass reductions and injects a level of risk-reduction into the design process. A similar open/closed-loop dynamic is used for load cases 3 and 4 , at $-1 g$. Load case 5 is a $1 g$ cruise case, only included to facilitate gust loads in the following sections. The final load case 6 is a simple $2 g$ inertial-only landing load, and is not aeroelastically coupled.

Table 1. Summary of static aeroelastic load cases.

\begin{tabular}{rrrrrr} 
Case & $N$ & Mach & Altitude & Safety Factor & Actuator Status \\
\hline 1 & 2.5 & 0.64 & $0 \mathrm{ft}$ & 1.5 & closed-loop \\
2 & 2.5 & 0.64 & $0 \mathrm{ft}$ & 1.3 & open-loop \\
3 & -1 & 0.64 & $0 \mathrm{ft}$ & 1.5 & closed-loop \\
4 & -1 & 0.64 & $0 \mathrm{ft}$ & 1.3 & open-loop \\
5 & 1 & 0.85 & $35,000 \mathrm{ft}$ & 1.5 & open-loop \\
6 & 2 & - & - & 1.5 & open-loop
\end{tabular}

\section{Dynamic Aeroelastic Modeling}

The dynamic aeroelastic behavior of the wing is modeled in the time domain (state-space), via modal coordinates: $\boldsymbol{u}=\boldsymbol{\phi} \cdot \boldsymbol{\eta}$, where $\boldsymbol{\phi}$ is a matrix of mode shapes, and $\boldsymbol{\eta}$ is a vector of modal amplitudes. Rigid body modes are included, but only symmetric motion: hence the first two modes of $\phi$ are pitch and plunge. The equations of motion are:

$$
\dot{\boldsymbol{x}}=\boldsymbol{A} \cdot \boldsymbol{x}+\boldsymbol{B}_{\theta} \cdot \boldsymbol{\theta}+\boldsymbol{B}_{\delta} \cdot \delta+\boldsymbol{B}_{w} \cdot w
$$

The dynamic aeroelastic solution vector $\boldsymbol{x}$ contains the modal amplitudes, $\boldsymbol{\eta}$, their time derivatives, $\dot{\boldsymbol{\eta}}$, aerodynamic lag states via a Roger approximation, ${ }^{21}$ control surface aerodynamic terms and their lag states, third-order actuator modeling terms, gust column aerodynamic lag terms, and gust forming filter terms. The control surface deflection vector $\boldsymbol{\theta}$ is retained from the previous section (though the actual control surface deflection experienced by the wing will be attenuated via the third-order actuator models), as is the elevator deflection $\delta$. Finally, $w$ is a white noise input meant to emulate a gust signal, which is sent through a forming filter embedded within $\boldsymbol{A}$. Additional details concerning Eq. 6 can be found in Refs. 7, 11, and 16.

A simple FCS is implemented via the equation $\delta=-\boldsymbol{K}_{\delta} \cdot \boldsymbol{x}$, which drives the elevator motion based on proportional control from the vehicle rigid body pitch and pitch rate. The terms within $\boldsymbol{K}_{\delta}$ are held fixed throughout all of the optimization results presented here.

\section{A. Flutter Constraints}

For the derivation of flutter constraints, AFS is not considered (i.e., $\boldsymbol{\theta}=\mathbf{0}$ ). Eigenvalues of $\overline{\boldsymbol{A}}=\boldsymbol{A}-\boldsymbol{B}_{\delta} \cdot \boldsymbol{K}_{\delta}$ are given by $s=g+i \cdot \omega$. For a given Mach number, these eigenvalues may be tracked across a range of matched point equivalent air speeds. Speeds at which a given eigenvalue crosses into the right-half plane $(g=0)$ are flutter points. A flutter constraint is formulated by imposing upper limits on the damping $g$ of 
each mode, at all equivalent air speeds $\left(U_{E A S}\right)$ of interest. ${ }^{22}$ Specifically, each damping value is constrained to lie beneath the piecewise polynomial:

$$
g \leq \begin{cases}0 & 0 \leq U_{E A S} \leq U^{*} \\ c \cdot\left(U_{E A S}-U^{*}\right)^{2} & U_{E A S}>U^{*}\end{cases}
$$

The minimum allowable flutter equivalent air speed is $U^{*}$, computed from the desired flutter margin (1.15) and the flight envelope (using a $185 \mathrm{~m} / \mathrm{s}$ dive speed). The quadratic scaling parameter $c$ is set to 500 . Critical $U_{E A S}$ points (local minima) of the inequality in Eq. 7 are computed and lumped together into a single KS constraint. As above, if this constraint is greater than one, then Eq. 7 is not satisfied, and the structure does not meet the required flutter margin.

\section{B. Gust Constraints}

The state covariance matrix, $\boldsymbol{X}$, due to a gust input, is computed via a Lyapunov equation:

$$
\overline{\boldsymbol{A}} \cdot \boldsymbol{X}+\boldsymbol{X} \cdot \overline{\boldsymbol{A}}^{T}+\overline{\boldsymbol{Q}}=\mathbf{0}
$$

where $\overline{\boldsymbol{Q}}=\boldsymbol{B}_{w} \cdot U_{\sigma}^{2} \cdot \boldsymbol{B}_{w}^{T}$. The design gust velocity, $U_{\sigma}$, is taken here to be $25.9 \mathrm{~m} / \mathrm{s} .{ }^{17,23}$ Having computed the covariance $\boldsymbol{X}$, it is desired to compute the $3 \times 3$ stress covariance matrix (in terms of $\sigma_{x}, \sigma_{y}$, and $\sigma_{x y}$ ) for each finite element, and the $2 \times 2$ traction covariance matrix (in terms of $N_{x}$ and $N_{x y}$ ) for each stiffened panel. Attempting to compute these via a transformation based upon $\boldsymbol{B}_{\sigma_{e}} \cdot \boldsymbol{\phi} \cdot \boldsymbol{\eta}$ will not work, since stresses are not easily extracted from modal quantities. Instead, modal acceleration (MA) must be used, a technique exhaustively described in Ref. 16, in the context of gust loads. Only cursory details will be given here.

The modally-reduced elastic, inertial, and aerodynamic terms that constitute the aeroservoelatic equations of motion in Eq. 6 can be alternatively written in the Laplace domain:

$$
\left(s^{2} \cdot \overline{\boldsymbol{M}}^{s s}+s \cdot \overline{\boldsymbol{C}}^{s s}+\overline{\boldsymbol{K}}^{s s}-q \cdot \overline{\boldsymbol{A}}^{s s}\right) \cdot \boldsymbol{\eta}+\left(s^{2} \cdot \overline{\boldsymbol{M}}^{s c}-q \cdot \overline{\boldsymbol{A}}^{s c}\right) \cdot \boldsymbol{\Theta}-q \cdot \overline{\boldsymbol{A}}^{s e} \cdot \delta=q \cdot \overline{\boldsymbol{A}}^{s g} \cdot w_{g} / U
$$

where $\boldsymbol{M}, \boldsymbol{C}, \boldsymbol{K}$, and $\boldsymbol{A}$ are the mass, damping, stiffness, and aerodynamic matrices, respectively, and the overbar designates a modally-reduced quantity. $q$ is the dynamic pressure, $\Theta$ is the control surface rotation (related to $\boldsymbol{\theta}$ via a series of third order actuator transfer functions), and $w_{g}$ is the speed of the gust column, related to $w$ via a forming filter. The $s s, s c, s e$, and $s g$ superscripts designate structure-on-structure, control-on-structure, elevator-on-structure, and gust-on-structure partitioning.

For MA, the modally-reduced matrices in Eq. 9 must be partially un-projected back into the full-order finite element space. The columns of these matrices can remain in modal space, but the rows must be in the full-order finite element space. These new matrices may be designated as $\boldsymbol{M}^{s s} \cdot \boldsymbol{\phi}$, etc., where $\boldsymbol{M}^{s s}$ is the full-order mass matrix. The full-order structural deformation vector can then be computed as:

$$
\begin{aligned}
\boldsymbol{u} & =\left(\boldsymbol{K}^{s s}\right)^{-1} \\
& \cdot\left(-\left(s^{2} \cdot \boldsymbol{M}^{s s}+s \cdot \boldsymbol{C}^{s s}-q \cdot \boldsymbol{A}^{s s}\right) \cdot \boldsymbol{\phi} \cdot \boldsymbol{\eta}-\left(s^{2} \cdot \boldsymbol{M}^{s c}-q \cdot \boldsymbol{A}^{s c}\right) \cdot \boldsymbol{\Theta}+q \cdot \overline{\boldsymbol{A}}^{s e} \cdot \delta+q \cdot \boldsymbol{A}^{s g} \cdot w_{g} / U\right)
\end{aligned}
$$

Converting Eq. 10 back from the Laplace domain into the state-space terms used for Eq. 6, leads to a MA equation of the form:

$$
\boldsymbol{u}=\boldsymbol{H} \cdot \boldsymbol{x}
$$

where $\boldsymbol{H}$ involves solving a system of equations of the full-order stiffness matrix $\boldsymbol{K}^{s s}$, with the number of right-hand-sides equal to the size of the aeroservoelastic solution vector $\boldsymbol{x}$. The $3 \times 3$ stress covariance matrix of each finite element is then given by:

$$
\boldsymbol{C}_{\sigma_{e}}=\boldsymbol{B}_{\sigma_{e}} \cdot \boldsymbol{H} \cdot \boldsymbol{X} \cdot \boldsymbol{H}^{T} \cdot \boldsymbol{B}_{\sigma_{e}}^{T}
$$

A similar $2 \times 2$ traction covariance matrix can be computed for the buckling of each stiffened panel. 
Stresses are assumed to be the sum of $1 g$ loads (computed via load case 5 in Table 1 ) and the gust loads:

$$
\left\{\begin{array}{c}
\sigma_{x} \\
\sigma_{y} \\
\sigma_{x y}
\end{array}\right\}_{e}=\boldsymbol{s}_{e}=\boldsymbol{s}_{e_{1}}+\boldsymbol{s}_{e_{g}}
$$

An equal-probability hyper-surface is then written as:

$$
\boldsymbol{s}_{e_{g}}^{T} \cdot\left(\boldsymbol{C}_{\sigma_{e}}\right)^{-1} \cdot \boldsymbol{s}_{e_{g}}=1
$$

If the surface of Eq. 14 lies entirely within the failure envelope of Eq. 4, then the gust loads are considered acceptable. The critical point along the hyper-surface can be found with a method developed in Ref. 17, and involves solving the following constrained optimization problem:

$$
\text { maximize: } \quad \bar{g}_{e}=\boldsymbol{s}_{e}^{T} \cdot \boldsymbol{F} \cdot \boldsymbol{s}_{e}+\boldsymbol{f} \cdot \boldsymbol{s}_{e}-1, \quad \text { such that: } \quad\left(\boldsymbol{s}_{e}-\boldsymbol{s}_{e_{1}}\right)^{T} \cdot\left(\boldsymbol{C}_{\sigma_{e}}\right)^{-1} \cdot\left(\boldsymbol{s}_{e}-\boldsymbol{s}_{e_{1}}\right)=1
$$

If the optimal $\bar{g}_{e}$ value is less than 0 , then the gust-stress constraint is satisfied. A graphical example of this is shown in Fig. 3 for a two-dimensional case.

$\bar{g}_{e}$ is computed for each shell finite element along the wingbox, and then compressed into a single KS aggregation function. Static stress values in the previous section had been compressed into 13 separate KS functions, but due to the large computational costs of computing the adjoint design derivatives of the MA matrix, $\boldsymbol{H}$ (discussed in more detail below), only a single aggregation is considered here. This choice adds more conservatism into the KS approximation.

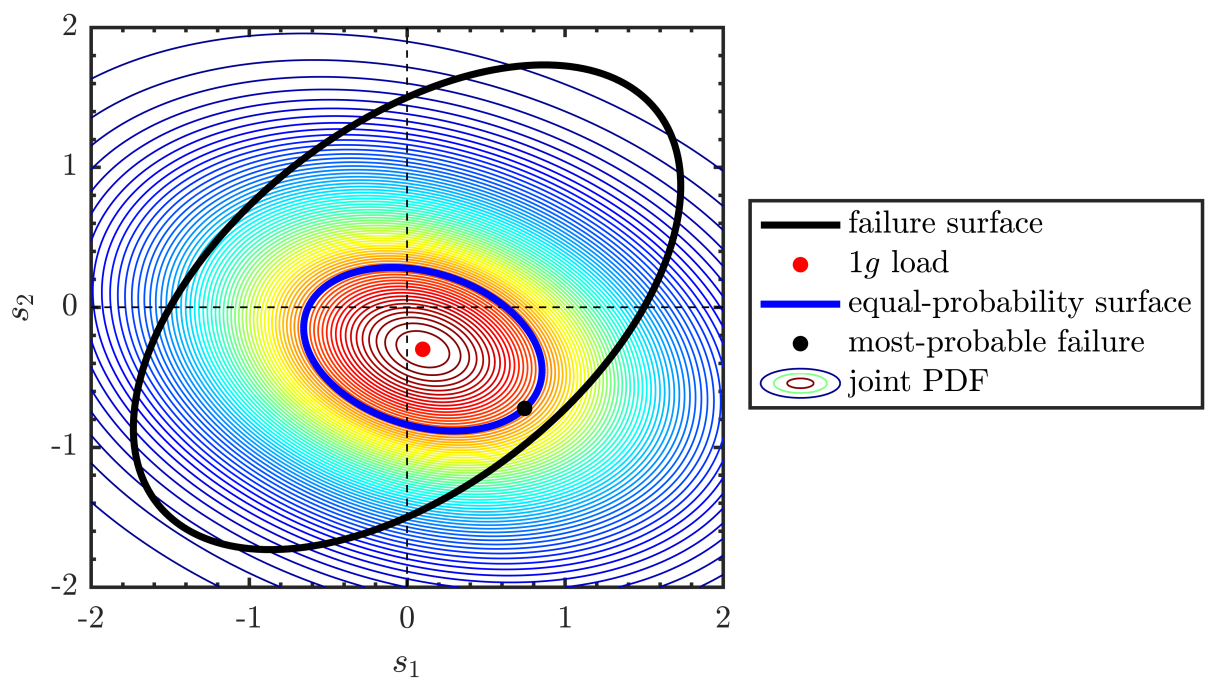

Figure 3. Two-dimensional example of an equal-probability hyper-surface and an elliptic failure boundary.

Similar methods to Eqs. 12-15 may be considered for skin buckling, and again wrapped into a single KS constraint. A significant approximation is made here, however, in that the buckling envelope $\left(N_{x, c r}\right.$ and $\left.N_{x y, c r}\right)$ used to build $\boldsymbol{F}$ and $\boldsymbol{f}$ in Eq. 15 is assumed to be static, independent of the loading frequency. This is known to be untrue ${ }^{24}$ but the inclusion of a load-dependent failure envelope is not easily included in the above framework, and is left for future work.

\section{Open-Loop Gust Constraints}

For open-loop analysis, the control surface rotations (commanded rotation $\boldsymbol{\theta}$ and actual rotation $\boldsymbol{\Theta}$ ) are set equal to 0 . The FCS controller driving the elevator motion $\delta$ remains active, and the safety factor associated with gust-based stress and buckling constraints is set to 1.5 . 


\section{Closed-Loop Gust Constraints}

For closed-loop analysis, both linear quadratic regulator (LQR) and linear quadratic Gaussian (LQG) controllers are used to drive the trailing edge control surfaces in this work. For LQR, a controller is assumed of the form $\boldsymbol{\theta}=-\boldsymbol{K}_{\boldsymbol{\theta}} \cdot \boldsymbol{x}$, and computed with the typical cost function: ${ }^{25}$

$$
J=\frac{1}{2} \cdot \int_{0}^{\infty}\left(\boldsymbol{x}^{T} \cdot \boldsymbol{Q}_{x} \cdot \boldsymbol{x}+\boldsymbol{\theta}^{T} \cdot \boldsymbol{R}_{\theta} \cdot \boldsymbol{\theta}\right) \cdot d t
$$

where $\boldsymbol{Q}_{x}$ is the state weighting matrix, and $\boldsymbol{R}_{\theta}$ is the control weighting matrix. The gust constraint process described above is the same, with the exception that now Eq. 8 uses $\overline{\boldsymbol{A}}=\boldsymbol{A}-\boldsymbol{B}_{\delta} \cdot \boldsymbol{K}_{\delta}-\boldsymbol{B}_{\theta} \cdot \boldsymbol{K}_{\theta}$. The MA matrix, $\boldsymbol{H}$, is identical to the previous section, since the state vector $\boldsymbol{x}$ is independent of the commanded control surface rotations $\boldsymbol{\theta}$, but only a function of the actual control surface rotations $\boldsymbol{\Theta}$.

LQR assumes full-state feedback, which is rarely available. LQG assumes the presence of an output based on actual sensors: $\boldsymbol{y}=\boldsymbol{C}_{x} \cdot \boldsymbol{x}+\boldsymbol{D}_{\theta} \cdot \boldsymbol{\theta}+\boldsymbol{D}_{\delta} \cdot \delta+\boldsymbol{v}$, where $\boldsymbol{v}$ is the sensor noise. For this work, six triaxial accelerometers are distributed along the wingbox, six strain gage rosettes are distributed along the upper skins, and six rosettes along the lower skins. The relevant $\overline{\boldsymbol{A}}$ matrix is now augmented by observer terms:

$$
\overline{\boldsymbol{A}}=\left[\begin{array}{cc}
\boldsymbol{A}-\boldsymbol{B}_{\delta} \cdot \boldsymbol{K}_{\delta}-\boldsymbol{B}_{\theta} \cdot \boldsymbol{K}_{\theta} & \boldsymbol{B}_{\delta} \cdot \boldsymbol{K}_{\delta}+\boldsymbol{B}_{\theta} \cdot \boldsymbol{K}_{\theta} \\
\mathbf{0} & \boldsymbol{A}-\boldsymbol{L} \cdot \boldsymbol{C}_{x}
\end{array}\right]
$$

where $\boldsymbol{L}$ is the Kalman gain, computed with a similar cost function as in Eq. 16, but with the state weighting $\boldsymbol{Q}_{x}$ replaced with the process noise matrix $\boldsymbol{B}_{w} \cdot U_{\sigma}^{2} \cdot \boldsymbol{B}_{w}^{T}$ from Eq. 8, and the control weighting $\boldsymbol{R}_{\theta}$ replaced with the covariance matrix of the sensor noise $\boldsymbol{v}$, designated as $\boldsymbol{R}_{v}{ }^{25}$ The relevant $\overline{\boldsymbol{Q}}$ matrix is now:

$$
\overline{\boldsymbol{Q}}=\left[\begin{array}{cc}
\boldsymbol{B}_{w} & \mathbf{0} \\
\boldsymbol{B}_{w} & -\boldsymbol{L}
\end{array}\right] \cdot\left[\begin{array}{cc}
U_{\sigma}^{2} & \mathbf{0} \\
\mathbf{0} & \boldsymbol{R}_{v}
\end{array}\right] \cdot\left[\begin{array}{cc}
\boldsymbol{B}_{w} & \mathbf{0} \\
\boldsymbol{B}_{w} & -\boldsymbol{L}
\end{array}\right]^{T}
$$

Using these new definitions of $\overline{\boldsymbol{A}}$ and $\overline{\boldsymbol{Q}}$ in Eqs. 17 and 18, the state covariance matrix $\boldsymbol{X}$ may be computed via Eq. 8, where $\boldsymbol{X}$ has twice the number of states as for the LQR case, or the un-controlled case, due to the observer terms. These observer terms have no impact on the modal acceleration process, and so the MA matrix must simply be augmented by a zero-matrix: $\left[\begin{array}{ll}\boldsymbol{H} & \mathbf{0}\end{array}\right]$. The remaining MA process of Eqs. $12-15$ is the same as above.

If GLA is to be considered in the optimization process, closed-loop gust constraints (stress and buckling) are augmented with a safety factor of 1.5. Similar to the methods used for static MLA, an open-loop gust constraint is retained, albeit at a lower safety factor of 1.3 .

\section{Optimization Definition}

The gradient based optimization problem solved here is given in Table 2. The objective function to be minimized is the structural wingbox mass. Design variables $\boldsymbol{q}$ include sizing variables, MLA rotation variables, and control law variables. For sizing, the wingbox is broken into 283 design patches, where each rib-delineated skin panel is a patch, each rib-delineated spar section is a patch, and each rib is a patch. Within each patch, the thickness of each shell member ( $t$, ranging from 3 to $30 \mathrm{~mm}$ ), the thickness of the stiffeners attached to these shells $\left(t_{s}\right.$, ranging from 2.5 to $\left.30 \mathrm{~mm}\right)$, and the height of the stiffeners $\left(h_{s}\right.$, ranging from 30 to $100 \mathrm{~mm}$, except in the rib and spar webs, where the lower bound is $64 \mathrm{~mm}$ ), are independently optimized. For this work, the height of each T-stiffener web is set equal to the flange width, and the thickness of the web and flange are equal as well, as drawn in Fig. 4. MLA rotation design variables include the vector of commanded control surface rotations, $\boldsymbol{\theta}$, for the closed-loop maneuver load cases 1 and 3 in Table 1, bounded by $\pm 10^{\circ}$. The control law design variables are the members of the diagonal control weighting matrix $\boldsymbol{R}_{\theta}$ (one variable per control surface), bounded between $10^{-2}$ and $10^{12}$.

Design constraints are also listed in Table 2, and include the static stress constraints $K S_{\sigma}$, where $N_{\sigma}$ is the number of aggregation parameters (set to 13 as noted above), and $N_{L}$ is the number of load cases (6 in Table 1). Similarly, $K S_{\mu}$ are the static buckling constraints, with $N_{\mu}$ set to 8 . A flutter constraint (Eq. 7), open- and closed-loop stress-based gust constraints, and open- and closed-loop buckling-based gust constraints (Eq. 15) are also included. As a surrogate on controller cost, the RMS rotation rate for each control surface during closed-loop GLA is computed via the covariance matrix $\boldsymbol{X}$, aggregated into a single KS 
Table 2. Optimization definition.

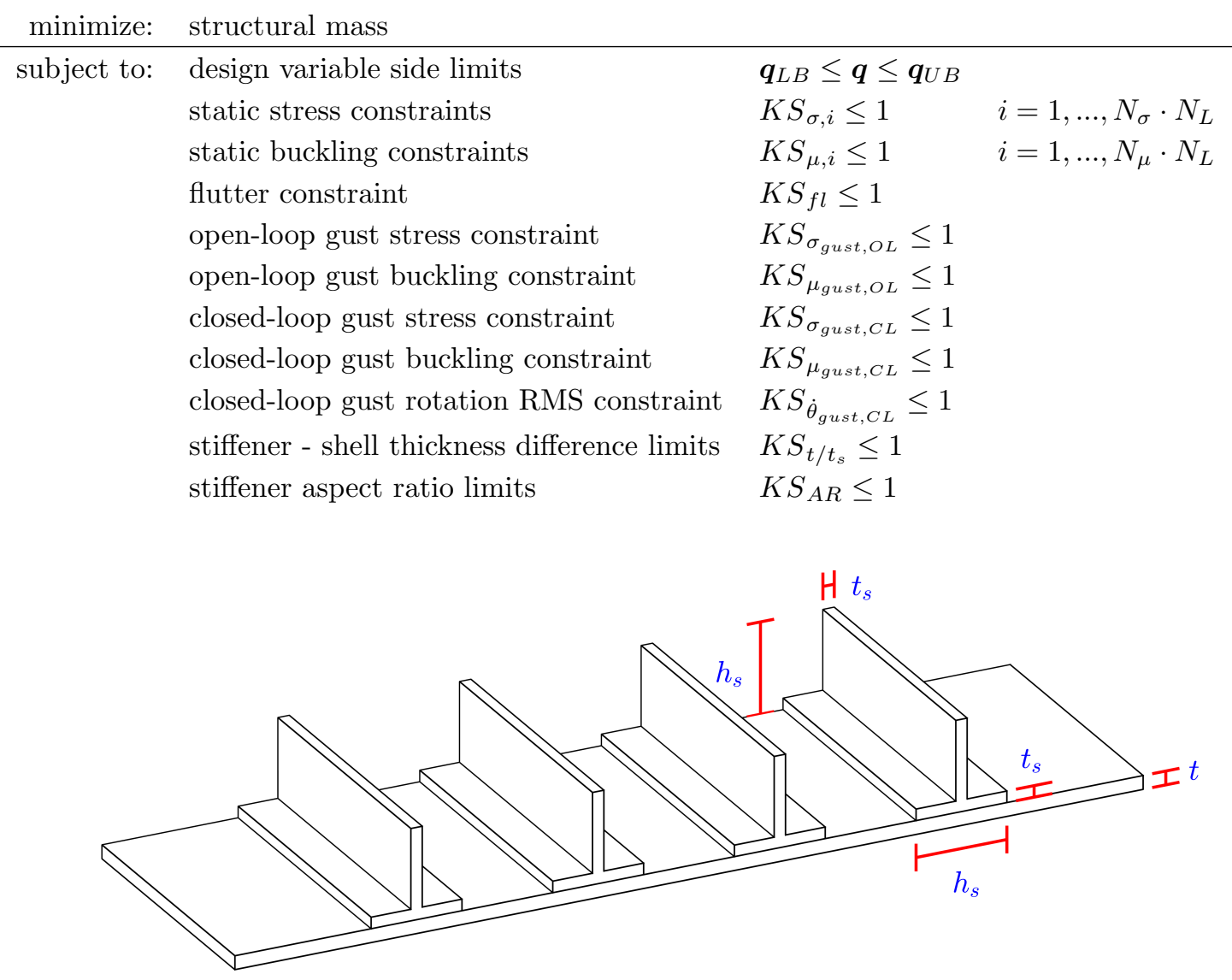

Figure 4. Example T-stiffener geometry.

constraint $K S_{\dot{\theta}_{\text {gust }, C L}}$, and forced to be less than some threshold. ${ }^{2}$ Ref. 26 provides guidance on reasonable ratios between rate saturation and the allowable RMS value (i.e., three standard-deviations): a range of limits are explored here. Furthermore, the same rotation rate limits are imposed on each control surface, an over-simplification given the range of actuator sizes used from root to tip.

The final two constraints in Table 2 regulate the stiffener geometry. It is desired that, for a given design patch, the shell thickness be within $2.5 \mathrm{~mm}$ of the stiffener thickness. These constraints are normalized to 1 for each design patch, and then all aggregated into a single metric, $K S_{t / t_{s}}$. Similarly, the aspect ratio of each stiffener should not be greater than 15, constraints which are normalized to 1 and then compressed into a single constraint, $K S_{A R}$.

Each set of design variables is passed through a linearly-decaying cone-shape filter ${ }^{27}$ in order to prevent the difference in stiffness between adjacent patches, or the difference in actuation between adjacent control surfaces, from being too large. The gradient-based optimization problem of Table 2 is solved with the Globally-Convergent Method of Moving Asymptotes tool (GCMMA). ${ }^{28}$ Design derivatives of the static aeroelastic system in Eq. 1 are computed with the adjoint method, and derivatives of the flutter constraint in Eq. 7 are computed with eigenvalue sensitivity analysis methods. ${ }^{29}$ Derivatives of the gust constraints are substantially more complex. Starting with Eq. 15, four design variable dependencies must be included in the chain rule:

- Derivatives of $\bar{g}_{e}$ with respect to the covariance matrix $\boldsymbol{X}$, and explicit derivatives of $\boldsymbol{X}$ with respect to the design variables $\boldsymbol{q}$. The latter involves differentiating the Lyapunov equation, and for closed-loop cases, derivatives of the LQR or LQG controllers must also be included. ${ }^{30}$

- Derivatives of $\bar{g}_{e}$ with respect to the MA matrix $\boldsymbol{H}$, and explicit derivatives of $\boldsymbol{H}$ with respect to the design variables $\boldsymbol{q}$. The derivatives of $\boldsymbol{H}$ are computed via the adjoint method, which involves solving 
a system of equations on the stiffness matrix $\boldsymbol{K}^{s s}$, with the number of right-hand-sides equal to the size of the covariance matrix $\boldsymbol{X}$ multiplied by the number of KS constraints. Given the large cost of this operation, only a single KS constraint is included for stress and buckling in Table 2, as noted previously.

- Derivatives of $\bar{g}_{e}$ with respect to the $1 g$ loads $\boldsymbol{s}_{e_{1}}$, computed via an adjoint computation of Eq. 1 .

- For buckling only, derivatives of $\bar{g}_{e}$ with respect to the failure envelope $\boldsymbol{F}$ and $\boldsymbol{f}$, and the derivative of this envelope with respect to the sizing design variables.

Further details of the gust constraint sensitivity analysis process are outlined in Ref. 16 and 17.

\section{Results}

Aeroelastic optimization results are conducted in five stages. First, only static aeroelastic optimization of an un-sized baseline wingbox is considered, without MLA. Secondly, MLA-based design variables (i.e., the vector of control surface rotations, $\boldsymbol{\theta}$ ) are included. Third, a flutter constraint is added. Fourth, open-loop gust constraints are added. Fifth, closed-loop gust constraints are added, as are the controller-based design variables (i.e., the members of the matrix $\boldsymbol{R}_{\theta}$ ). The convergence results of stages 1-4 are shown in Fig. 5, with and without MLA. Stage 1 converges to a structural wingbox mass of 18,666 kg; the optimal sizing parameters along the wingbox are shown in Fig. 6 , in terms of $t, t_{s}$, and $h_{s}$ of each design patch.

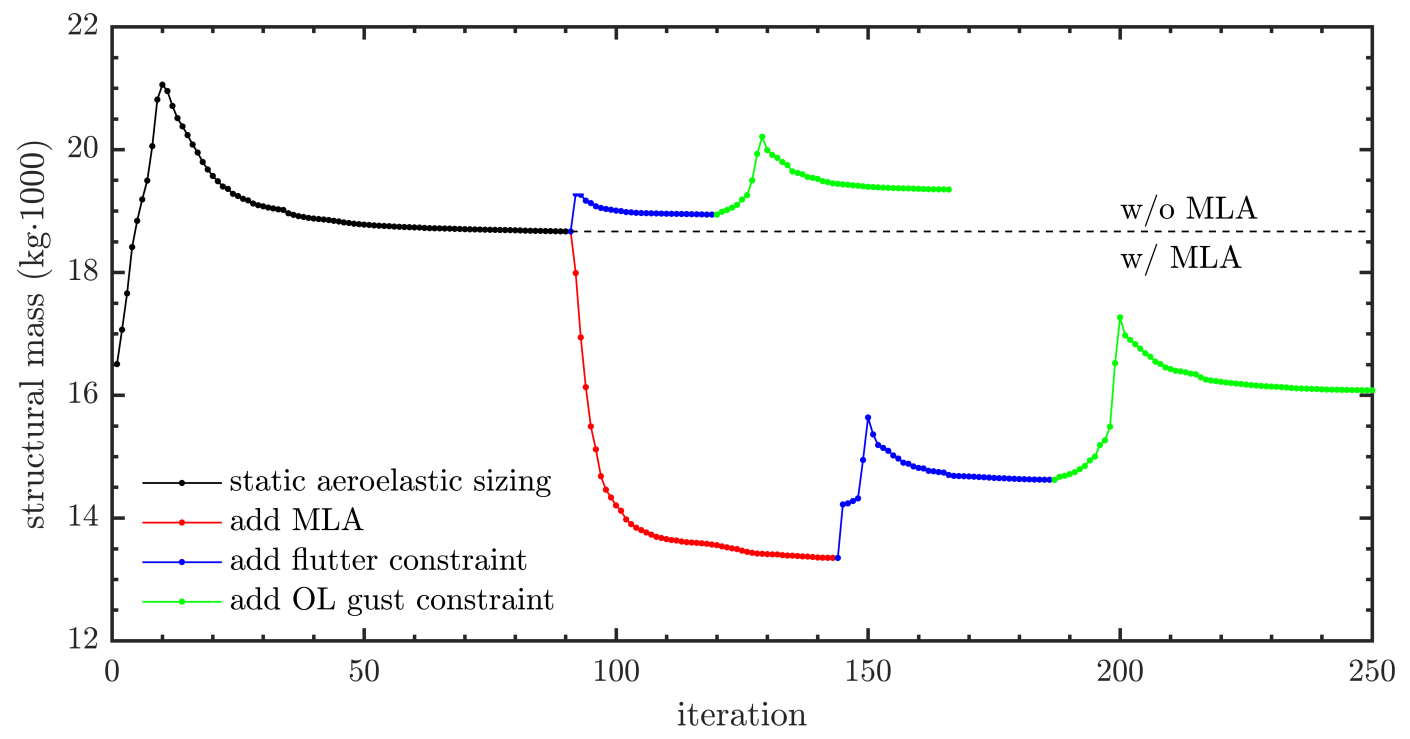

Figure 5. Optimization convergence with and without MLA.

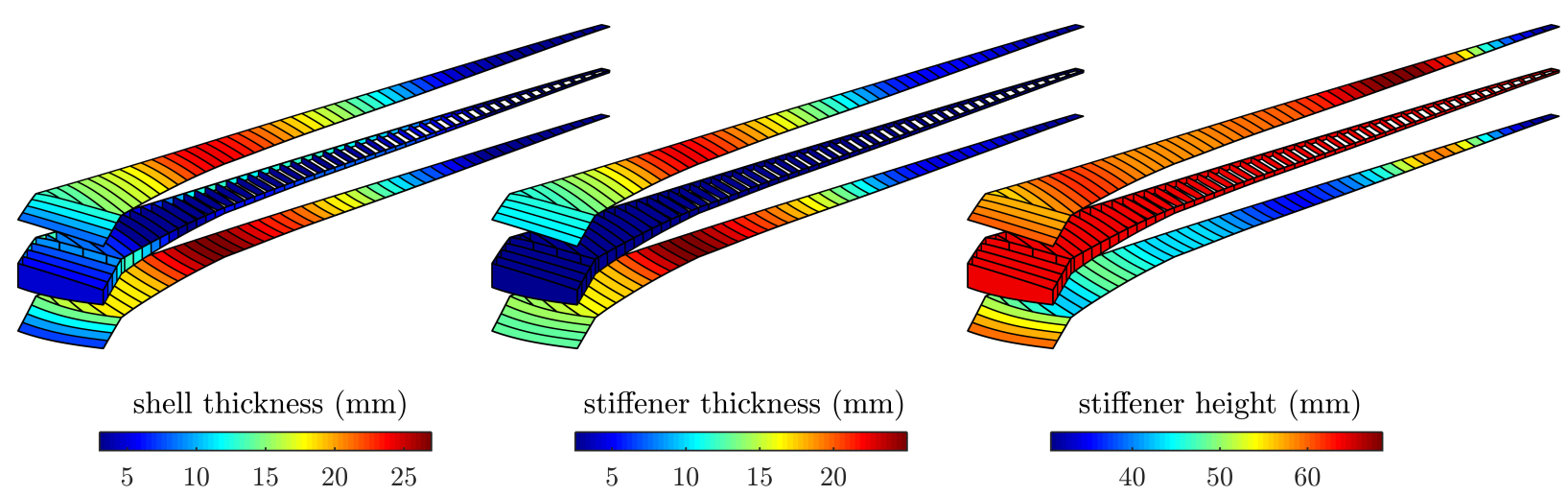

Figure 6. Optimization distribution of $t, t_{s}$, and $h_{s}$ along the wingbox. 
This first-stage aeroelastic sizing converges after 91 iterations; MLA design variables (lower portion of Fig. 5) are included betweens iterations 92 and 144. Structural mass decreases substantially, from 18,666 $\mathrm{kg}$ to $13,349 \mathrm{~kg}$, and the optimal control surface rotation variables for the closed-loop cases $1(2.5 \mathrm{~g})$ and 3 $(-1 g)$ are shown in Fig. 7. For the positive load case, MLA shifts the center of lift towards the root, with negative rotations (load alleviation) outboard for bending moment reductions, and positive rotations (load augmentation) inboard to help maintain trim. The optimal sizing variables are qualitatively similar to those seen in Fig. 6 (though thinner throughout), and are not shown. The mass reduction due to MLA is expected to be proportional to the open-loop safety factors, set to 1.3 here. A lower value will allow the optimizer to remove additional mass; a value of 1.5 (equal to that attached to the closed-loop cases) will revert the structural mass back to $18,666 \mathrm{~kg}$, which is the same value found by the optimizer without MLA included.

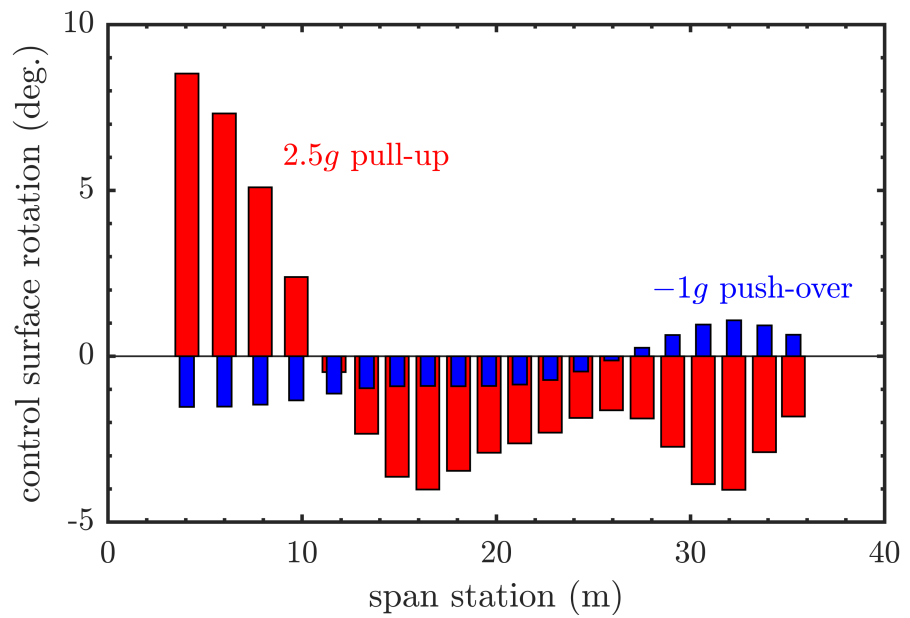

Figure 7. Optimal control surface rotations during the two closed-loop static aeroelastic maneuvers.

After the second-stage MLA results converge at iteration 144, a flutter constraint is added (increasing the structural mass to $14,622 \mathrm{~kg}$ ), and an open-loop gust constraint is subsequently added (increasing the structural mass to $16,078 \mathrm{~kg}$ ). These structural mass penalties due to dynamic aeroelastic constraints are substantial: relative to the static aeroelastic MLA result, the flutter constraint penalizes mass by $9.5 \%$, and the open-loop gust constraint by $20.4 \%$. This is in strong contrast to the results without MLA included (upper portion of Fig. 5), where the flutter and gust constraints only add $1.4 \%$ and $3.6 \%$, respectively. Only aeroelastic optimization with MLA included is considered for the remainder of the results in this paper.

Eigenvalue migration plots (real part only) of the third-stage design results are shown in Fig. 8, both before and after the addition of the flutter constraint in Eq. 7. This flutter constraint is highly active for the final feasible design on the right side of Fig. 8, with four roots strongly interacting with the constraint boundary: the phugoid mode, the second bending mode (exhibits a "hard" flutter mechanism), an inplane bending mode, and a torsional engine-pitch mode (both exhibiting softer "hump" flutter mechanisms). Finally, the open-loop buckling constraint is added to Fig. 5 for the fourth design stage. Flutter plots for this final design are very similar to those shown in Fig. 8, and are not given here. Gust-based buckling constraints $\left(\bar{g}_{e}\right.$ from Eq. 15$)$ are shown in Fig. 9.

The gust buckling constraint is less than 0 for all of the upper and lower skin panels throughout the wingbox, indicative of a feasible design. The constraint is in fact active, but the largest $\bar{g}_{e}$ is -0.21 , which is a result of the conservatism of the KS function. The equal-probability surface, failure surface, $1 g$ loads, and the most-probable failure point (i.e., the point which maximizes $\bar{g}_{e}$ in Eq. 15) are also shown in the figure, for three selected panels. The axial loads $N_{x}$ are clearly more prevalent than the shear loads $N_{x y}$ for both the steady $1 g$ case as well as the probabilistic gust covariance. Panel 66 (lower skins towards the wingtip) is a case where the gust loads alone would be large enough to cause a failure, but the stabilizing effect of the $1 \mathrm{~g}$ loads (namely the tensile stresses along the lower skins of the deformed wing) retains the equal-probability surface within the failure envelope. This is in contrast to panels 5 and 50 in Fig. 9 (upper skins at tip and root, respectively), where the gust loads and the trim loads are largely additive. Finally, it is repeated here that the failure boundaries of Fig. 9 are assumed to be static, when in fact there will be some dependence on the loading frequency, ${ }^{24}$ rendering this failure surface non-deterministic in addition to the gust response. 

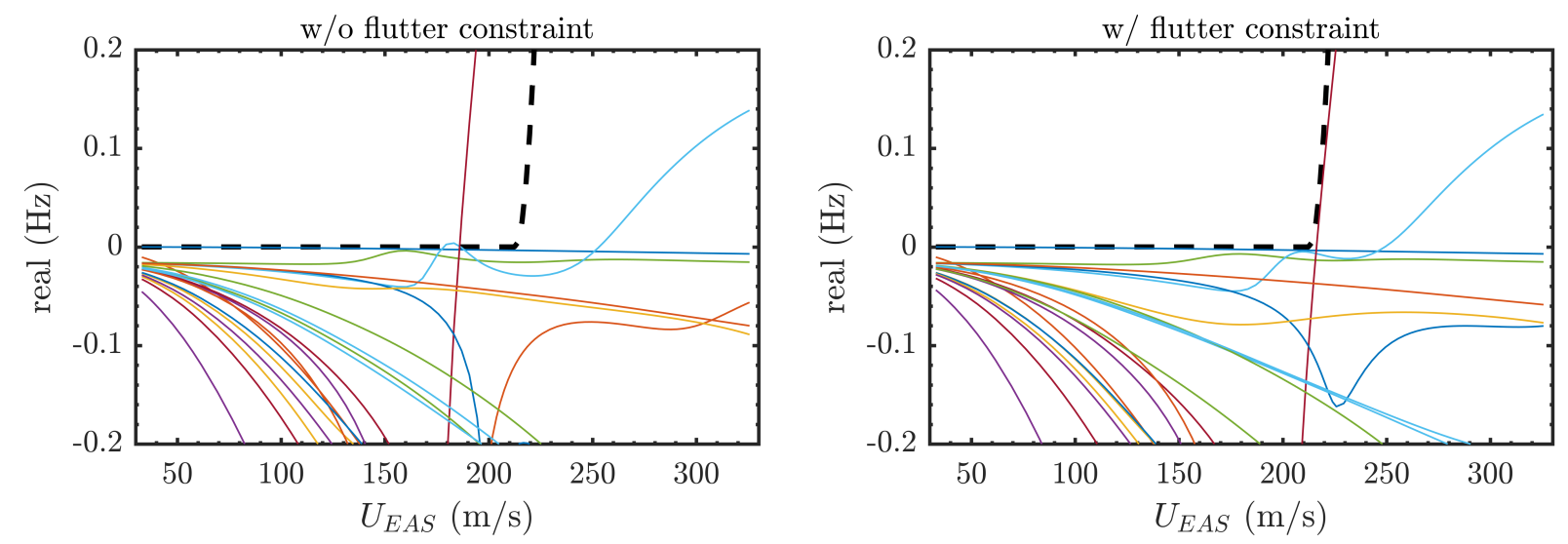

Figure 8. Eigenvalue migration (real part) before and after the flutter constraint is implemented; constraint boundary indicated by thick dashed line.

Additional insight into these buckling constraints is given in Fig. 10, which shows isolated contributions to the $\bar{g}_{e}$ constraint from trimmed $1 g$ loads only (by setting the covariance matrix to 0 ), from gust loads only (by setting the $1 g$ loads to 0 ), and the combined contribution. This final third plot in Fig. 10 of the combined contribution is reproduced from Fig. 9. Similar results are given for the gust-based stress constraints in Fig. 11. Stress-based failure and equal-probability surfaces for various points along the wingbox are not easily plotted (analogous to the results in Fig. 9), owing to the third dimension: both surfaces are threedimensional ellipsoids.

The complete gust constraints in Figs. 10 and 11 are not a linear sum of the individual trim and gust contributions, owing to the highly nonlinear operators involved in the solution to Eq. 15. For buckling, the trimmed $1 g$ constraint values are higher in the upper skins than lower, as the former is deformed in compression, and thus more buckling prone. Furthermore, these buckling constraints are nearly uniform along the upper surface, which is largely the result of the optimizer sizing each panel so as to remain free of buckling during the $2.5 \mathrm{~g}$ static loads. Though not shown here, this $2.5 \mathrm{~g}$ static buckling constraint is nearly active throughout the entire upper surface; the $1 g$ buckling constraint is nearly uniform as well (though of course not active). Gust constraints purely due to the gust covariance (middle drawing in Figs. 10) are concentrated outboard, presumably due to the high inertial loads. This leads to the aforementioned situation where pure gust-based buckling loads are infeasibly large; only the addition of the stabilizing $1 g$ loads produces a feasible combined response.

The dynamic stress constraints in Fig. 11 show similar trends to the buckling results: peak static $1 g$ stresses occur at the stress concentration near the trailing edge of the wing root. Combining this result with the dynamic gust loads helps push the high stress constraints outboard. The aggregate stress constraint is active in the final design, but again owing to the conservatism of the KS method, the highest stress-based $\bar{g}_{e}$ for any of the finite elements is only -0.2. Strategies to adaptively reduce the conservatism of KS constraints have been addressed (see Ref. 31, for example), but are not pursued here.

Design stages 1-4 are shown in Fig. 5: 1) initial static aeroelastic sizing, 2) MLA, 3) flutter constraints, and 4) open-loop gust constraints. The final stage is the introduction of closed-loop gust constraints. This is done by reducing the safety factor of the open-loop gust constraints (stress and buckling) from 1.5 to 1.3, adding closed-loop gust constraints (stress and buckling) with a safety factor of 1.5, adding a constraint on the maximum allowable control surface rotation rate RMS, ${ }^{2}$ and finally adding the control weighting design variables (members of $\boldsymbol{R}_{\theta}$ ). The optimal structural wingbox mass, across a range of RMS rate limits, is shown in Fig. 12, for both LQR and LQG controllers.

In addition to the LQR/LQG data, three additional structural mass values are indicated in Fig. 12, to bracket the data. First is the structural mass without any gust constraints, 14,622 kg, also shown in Fig. 5 at the end of the flutter-constrained optimization stage-3. Second is the structural mass when only an open-loop gust constraint is included with a safety factor of 1.5: 16,078 kg, also shown in Fig. 5 at the end of the gust-constrained stage- 4 . The prime goal here is to erase/recover this latter penalty through the use of GLA (AFS could also be used to recover the mass penalty arising from the flutter constraint, ${ }^{32}$ but this 

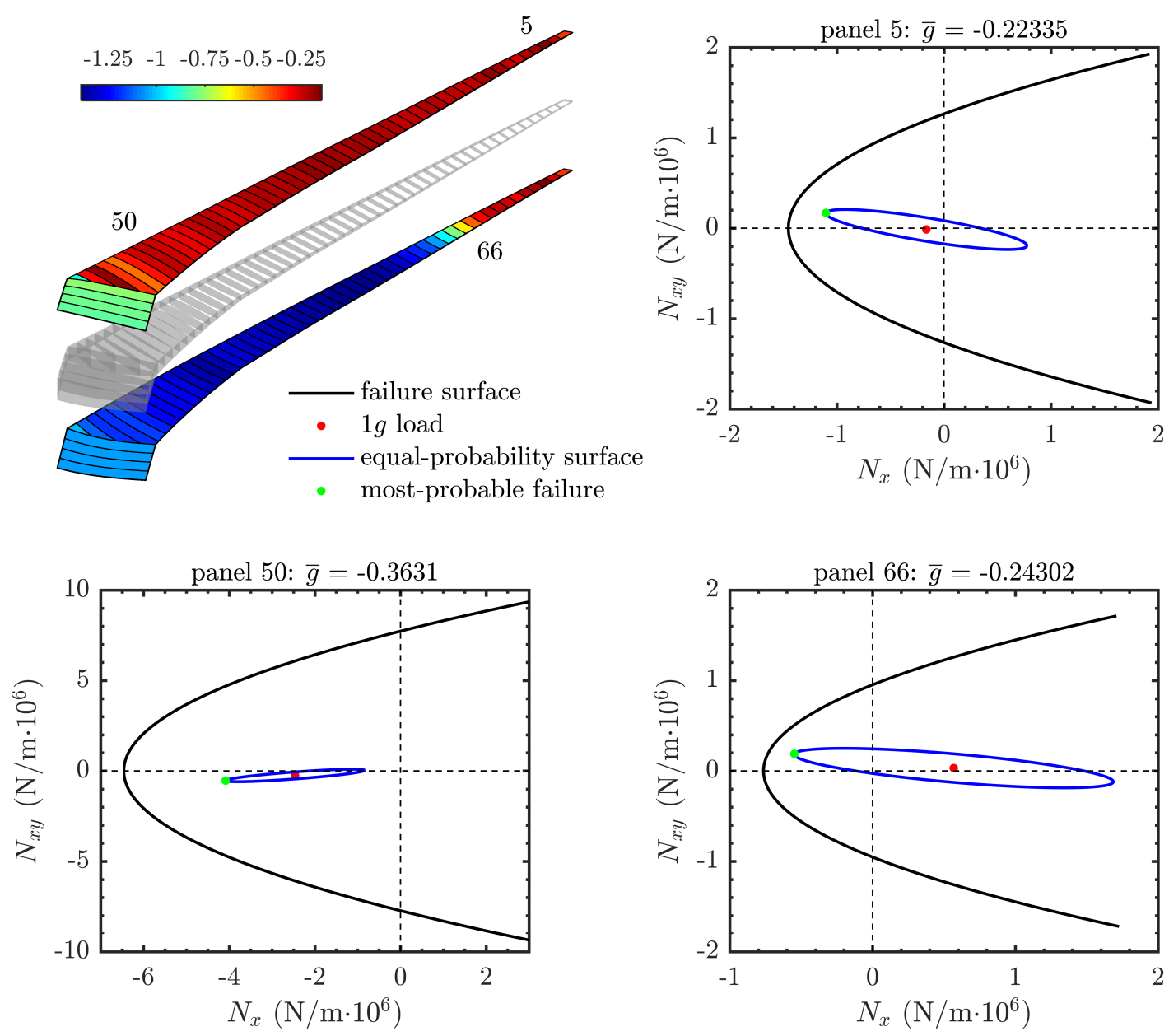

Figure 9. Open-loop gust-based buckling constraints along the paneled wingbox, and probabilistic failure boundaries for three selected panels.

is not considered here). The final mass value shown in Fig. 12 is the optimal structural mass when only an open-loop gust constraint with a safety factor of 1.3 is considered: $14,741 \mathrm{~kg}$. It can then be seen that the mass penalty when imposing an open-loop 1.5 gust constraint is substantial, but only a minor penalty arises from an open-loop 1.3 gust constraint. This last structural mass value of $14,741 \mathrm{~kg}$ is relevant because the GLA optimization cases (both LQR and LQG) converge to it, when large control surface rotation rates are allowed. In other words, if control cost is of no concern, the GLA controller is able to satisfy the closed-loop stress and buckling constraints entirely, without the need to stiffen the structure (and thus increase the structural mass). The wingbox can only satisfy the open-loop gust constraints using passive means.

Decreasing the allowed rotation rate RMS (i.e., making this constraint harder to satisfy), generally reduces the effectiveness of the GLA controller, and forces the closed-loop gust constraints to be partially borne by the wingbox itself, increasing the structural mass. For LQR, this begins to happen when the allowable rate RMS is forced below $50 \mathrm{deg}$./s. For RMS values below 2 deg./s, the LQR controller is completely ineffective, and the closed-loop gust constraints must be entirely satisfied by the wingbox. The optimal structural mass regresses to the same value found when only an open-loop gust constraint is included with a safety factor of 1.5: $16,078 \mathrm{~kg}$. In this limiting case, the closed-loop constraint has effectively been converted into an open-loop constraint.

It can also be seen in Fig. 12 that the LQG controller is less effective than its LQR counterpart. This is perhaps expected given that the LQG controller is driven by a finite amount of sensor data (triaxial accelerometers and strain gage rosettes), whereas LQR assumes full-state feedback. The LQR controller loses most of its effectiveness when the rotation rate RMS is forced below $2 \mathrm{deg}$./s, but this occurs for LQG at a much higher value of $30 \mathrm{deg}$./s. Of course, the LQG controller is defined by many parameters which 


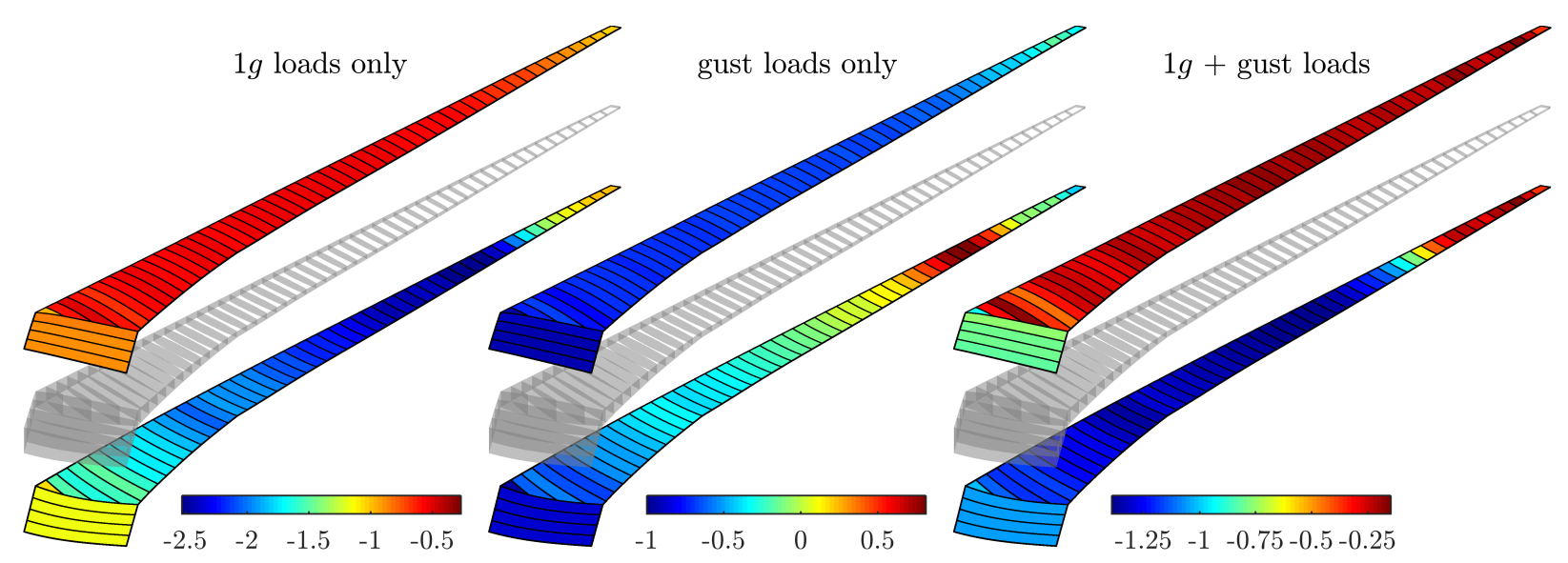

Figure 10. Open-loop gust-based buckling constraints along the paneled wingbox, with isolated contributions from trim loads (left) and gust loads (center).

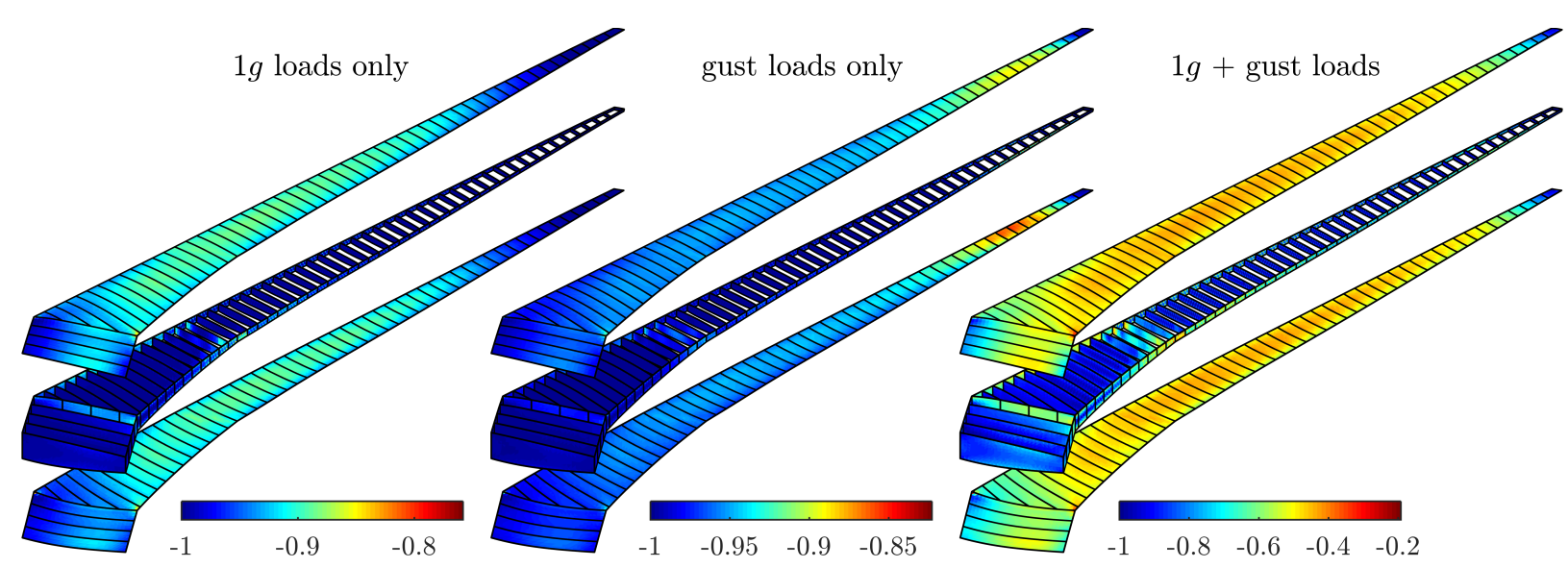

Figure 11. Open-loop gust-based stress constraints along the paneled wingbox, with isolated contributions from trim loads (left) and gust loads (center).

have not been fully explored here, including sensor noise $\boldsymbol{R}_{v}$ and the number/location/type of the sensors themselves. Different values for these parameters may change the LQG performance seen in Fig. 12.

The control surface rotations are shown for both LQR and LQG controllers in Fig. 13, for the case where there is no constraint on rotation rate RMS. As noted above, in this case both controllers are able to entirely satisfy the closed-loop gust constraints without the need to increase structural mass. Rotation rates are quite high as a result, with peak RMS values reaching 120 deg./s for the LQR controller, and 168 deg./s for LQG. The energy distribution of the two controllers is very different as well, with LQR concentrating high rotation rates towards the root (presumably driven by rigid body motions), while LQG is more evenly distributed over the wing. Though not included in the optimization in any way, control surface rotation angle RMS is shown in Fig. 13 as well, with the general trends mirroring the angular rate RMS values ${ }^{26}$ as expected. Elevator motions, driven by the FCS, are also shown in the figure, with the understanding that angular RMS values of the oscillating elevator would in reality be superimposed upon the steady elevator deflection needed for $1 g$ trim (which is not shown).

Finally, an extension of Fig. 13 is shown in Fig. 14, for the controlled cases where the peak RMS rate is limited to $40 \mathrm{deg}$./s. As with previous results, the largest rate RMS value seen in the figure is less than $40 \mathrm{deg} . / \mathrm{s}$, owing to the conservatism of the KS constraint. Unlike with the previous un-constrained result in Fig. 13, the constrained results in Fig. 14 demonstrate a less-smooth variation in control surface motion along the trailing edge: this is due to the optimizer's aggressive use of the control law design variables $\left(\boldsymbol{R}_{\theta}\right)$ in order to satisfy design constraints. These optimal values are not shown here, but can vary by orders of magnitude from the wing root to the tip. 


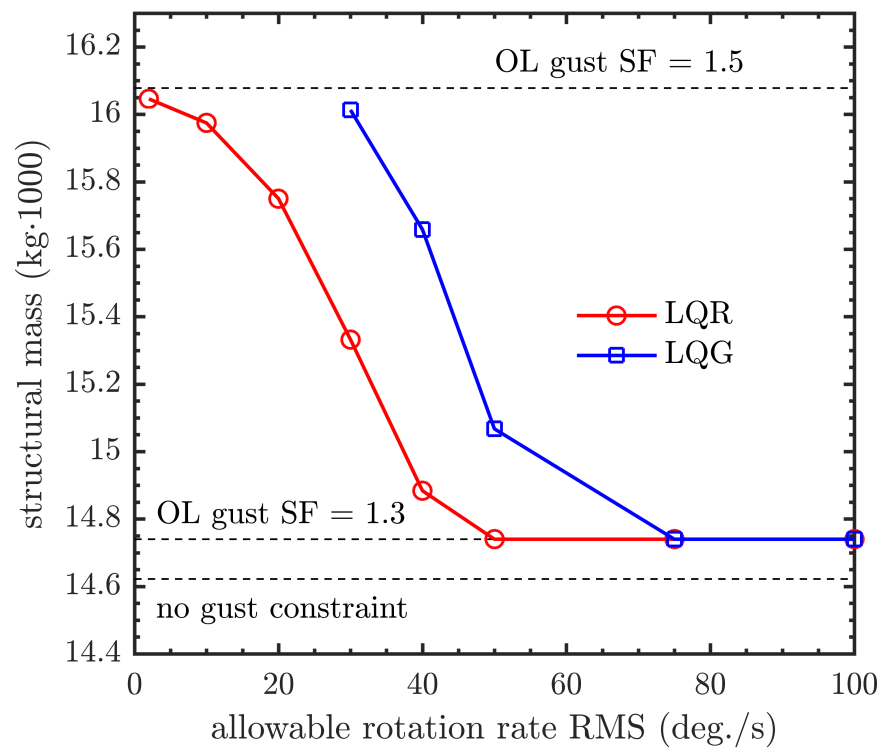

Figure 12. Structural mass penalty with reduced controller effectiveness.
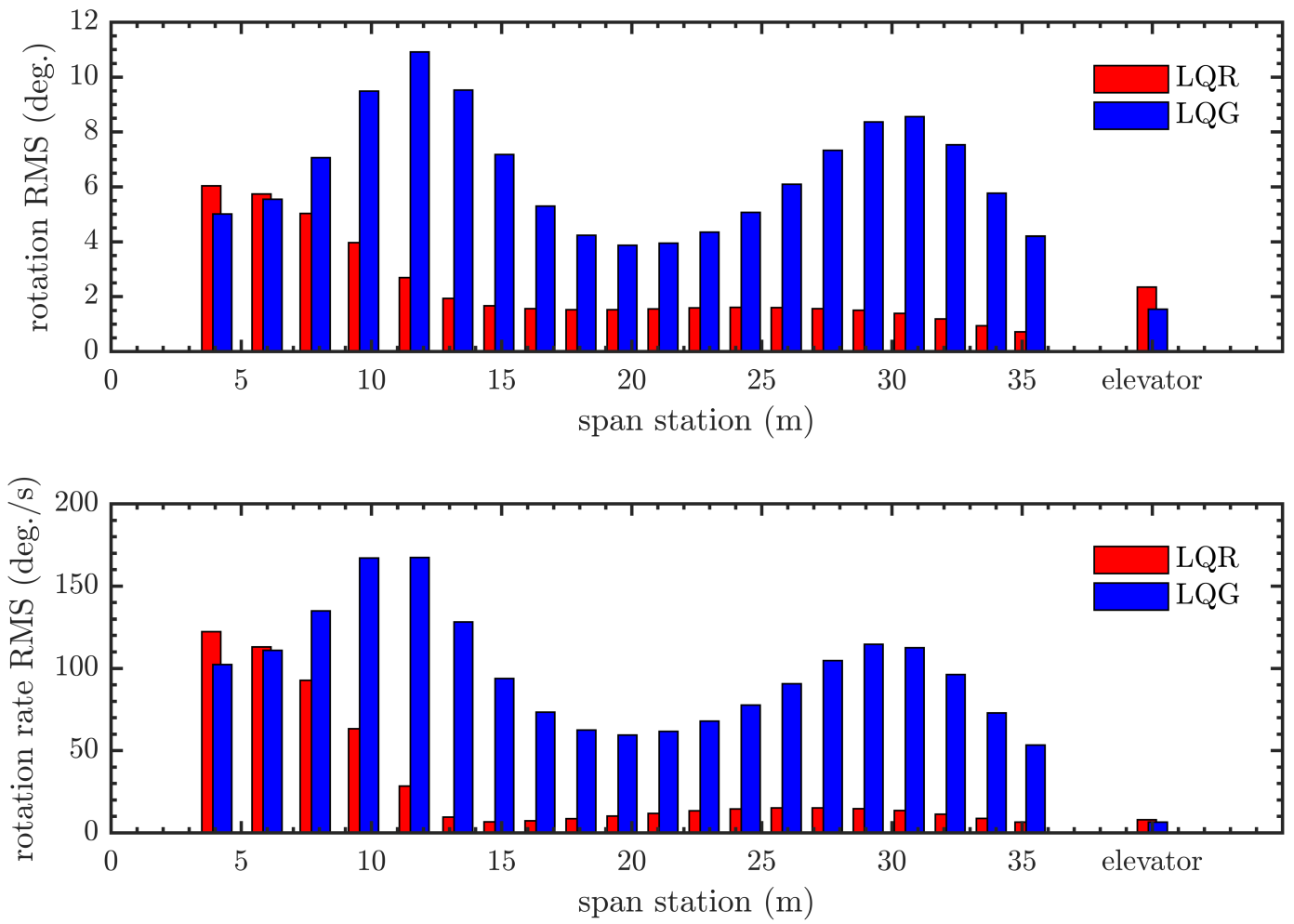

Figure 13. Control surface and elevator RMS motion for the case without limits on rate RMS.

\section{Conclusions}

This work has considered aeroservoelastic optimization of the Common Research Model transport configuration, with a standard rib/spar/skin/stiffener wingbox, and outfitted with several electrohydraulic control surfaces along the trailing edge. These control surfaces are used for both maneuver load alleviation and gust load alleviation. The objective function is to minimize structural mass, and design variables are set for 

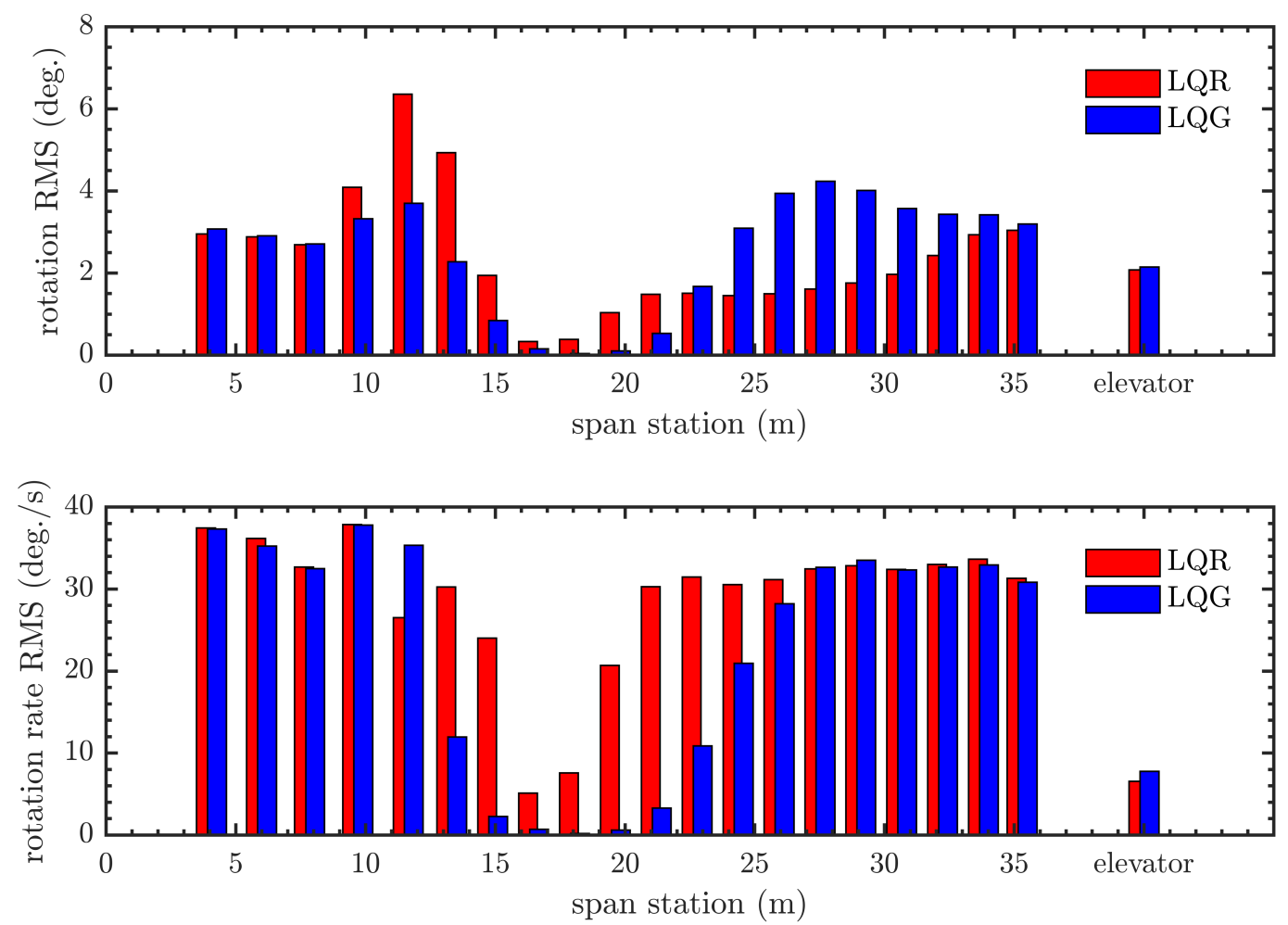

Figure 14. Control surface and elevator RMS motion for the case where rate RMS is limited to $40 \mathrm{deg} . / \mathrm{s}$.

detailed sizing of the wingbox members, steady control surface deflections during maneuver load alleviation, and distributed parameters used to define the closed-loop controllers for gust load alleviation. Aeroelastic design constraints include steady stress and buckling metrics across several trimmed maneuver loads, as well as flutter constraints, but the novel aspect of this paper is in the gust constraint formulation, which involves computing stress/buckling metrics in response to a stochastic continuous gust input. The formulation of these constraints echoes previous work done in Refs. 16 and 17, but is demonstrated here, for the first time, in the context of a complete design optimization for a realistic airplane configuration.

A summary of the various cases considered in this work is tabulated in Table 3. The dynamic aeroelastic constraints (flutter and gust) do not have a sizable impact on the problem if maneuver load alleviation is not considered, as the static stress and buckling constraints are stronger design drivers. Introducing maneuver load alleviation allows the optimizer to remove a significant amount of structural mass, thus rendering both flutter and gust as stronger drivers. If control cost is of no concern, the mass penalty introduced through the gust constraints can be largely recovered through closed-loop control. Handicapping the control surface rotation rates limits the effectiveness of the controllers, forcing the optimizer to add structural mass in order to (partially, at least) passively control the gust response. LQG controllers are more susceptible to this degradation than LQR, given that they are driven by a finite amount of sensor data.

\section{Acknowledgments}

This work is funded by the NASA Advanced Air Transport Technologies project. Thanks also to Dr. Frode Engelsen for his insight related to the problems considered here.

\section{References}

${ }^{1}$ Zeiler, T., Weisshaar, T., "Integrated Aeroservoelastic Tailoring of Lifting Surfaces," Journal of Aircraft, Vol. 25, No. 1, pp. 76-83, 1988.

${ }^{2}$ Livne, E., Schmidt, A., Friedmann, P., "Integrated Structure/Control/Aerodynamic Synthesis of Actively Controlled Composite Wings," Journal of Aircraft, Vol. 30, No. 3, pp. 387-394, 1993. 
Table 3. Summary of optimization cases.

\begin{tabular}{rrrrrrrr} 
case & MLA? & flutter margin & OL gust SF & CL gust SF & rate RMS & structural mass & figure \\
\hline 1 & no & - & - & - & - & $18,666 \mathrm{~kg}$ & Fig. 6 \\
2 & no & 1.15 & - & - & - & $18,939 \mathrm{~kg}$ & Fig. 5 \\
3 & no & 1.15 & 1.5 & - & - & $19,349 \mathrm{~kg}$ & Fig. 5 \\
4 & yes & - & - & - & - & $13,349 \mathrm{~kg}$ & Fig. 7 \\
5 & yes & 1.15 & - & - & - & $14.622 \mathrm{~kg}$ & Fig. 8 \\
6 & yes & 1.15 & 1.3 & - & - & $14,741 \mathrm{~kg}$ & Fig. 12 \\
7 & yes & 1.15 & 1.5 & - & - & $16,078 \mathrm{~kg}$ & Fig. 9 \\
8 & yes & 1.15 & 1.3 & 1.5 & no limit, LQR & $14,741 \mathrm{~kg}$ & Fig. 13 \\
9 & yes & 1.15 & 1.3 & 1.5 & no limit, LQG & $14,741 \mathrm{~kg}$ & Fig. 13 \\
10 & yes & 1.15 & 1.3 & 1.5 & 40 deg./s, LQR & $14,883 \mathrm{~kg}$ & Fig. 14 \\
11 & yes & 1.15 & 1.3 & 1.5 & 40 deg./s, LQG & $15,660 \mathrm{~kg}$ & Fig. 14
\end{tabular}

${ }^{3}$ Bindolino, G., Ricci, S., Mantegazza, P., "Integrated Servostructural Optimization in the Design of Aerospace Systems," Journal of Aircraft, Vol. 36, No. 1, pp. 167-175, 1999.

${ }^{4}$ Karpel, M., "Reduced-Order Models for Integrated Aeroservoelastic Optimization," Journal of Aircraft, Vol. 36, No. 1, pp. 146-155, 1999.

${ }^{5}$ Jackson, T., Livne, E., "Integrated Aeroservoelastic Design Optimization of Actively Controlled Strain-Actuated Flight Vehicles," AIAA Journal, Vol. 52, No. 6, pp. 1105-1123, 2014.

${ }^{6}$ Livne, E., "Integrated Aeroservoelastic Optimization: Status and Direction," Journal of Aircraft, Vol. 36, No. 1, pp. 122-145, 1999.

${ }^{7}$ Zole, A., Karpel, M., "Continuous Gust Response and Sensitivity Derivatives Using State-Space Models," Journal of Aircraft, Vol. 31, No. 5, pp. 1212-1214, 1994.

${ }^{8}$ Suzuki, S., Yonezawa, S., "Simultaneous Structure/Control Design Optimization of a Wing Structure with a Gust Load Alleviation System," Journal of Aircraft, Vol. 30, No. 2, pp. 268-274, 1993.

${ }^{9}$ Nam, C., Chattopadhyay, A., Kim, Y., "Optimal Wing Planform Design for Aeroelastic Control," AIAA Journal, Vol. 38, No. 8, pp. 1465-1470, 2000.

${ }^{10}$ Fidkowski, K., Engelsen, F., Willcox, K., Kroo, I., "Stochastic Gust Analysis Techniques for Aircraft Conceptual Design," AIAA Multidisciplinary Analysis and Optimization Conference, Victoria, Canada, September 10-12, 2008.

${ }^{11}$ Mor, M., Livne, L., "Sensitivities and Approximations for Aeroservoelastic Shape Optimization with Gust Response Constraints," Journal of Aircraft, Vol. 43, No. 5, pp. 1516-1527, 2006.

${ }^{12}$ Blelloch, P., "Calculation of Structural Dynamic Forces and Stresses using Mode Accleration," AIAA Journal, Vol. 12, No. 5, pp. 760-762, 1988.

${ }^{13}$ Livne, E., Blando, G., "Reduced Order Design-Oriented Stress Analysis using Combined Direct and Adjoint Solutions," AIAA Journal, Vol. 38, No. 5, pp. 898-909, 2000.

${ }^{14}$ Hajela, P., Bach, C., "Optimum Structural Sizing for Gust-Induced Response," Journal of Aircraft, Vol. 26, No. 4, pp. 395-397, 1989.

${ }^{15}$ de la Fuente, E., "Von Mises Stresses in Random Vibration of Linear Structures," Computers and Structures, Vol. 87, No. 21, pp. 1253-1262, 2009.

${ }^{16}$ Engelsen, F., Livne, E., "Modal Acceleration Based Random Gust Stresses in Aeroservoelastic Optimization," Journal of Aircraft, Vol. 41, No. 2, pp. 335-347, 2004.

${ }^{17}$ Engelsen, F., Livne, E., "Quadratic Stress Failure Constraints for Structures under Combined Steady and Random Excitation," AIAA Journal, Vol. 42, No. 1, pp. 132-140, 2004.

${ }^{18}$ Brooks, T., Kenway, G., Martins, J., "uCRM: An Aerostructural Model for the Study of Flexible Transonic Aircraft Wings," AIAA Journal, in press, 2018.

${ }^{19}$ Stroud, W., Agranoff, N., "Minimum-Mass Design of Filamentary Composite Panels under Combined Loads: Design Procedure Based on Simplified Buckling Equations," NASA TN D-8257, 1976.

${ }^{20}$ Kreisselmeier, G., Steinhauser, R., "Systematic Control Design by Optimizing a Vector Performance Index", International Federation of Active Controls Symposium on Computer-Aided Design of Control Systems, Zurich, Switzerland, 1979.

${ }^{21}$ Roger, K., "Airplane Math Modeling Methods for Active Control Design," AGARD CP-228, pp. 4-11, 1977.

${ }^{22}$ Ringertz, U., "On Structural Optimization with Aeroelasticity Constraints," Structural Optimization, Vol. 8, pp. 16-23, 1994.

${ }^{23}$ Hoblit, F., Gust Loads on Aircraft: Concepts and Applications, AIAA, Washington DC, 1988.

${ }^{24}$ Patel, S., Datta, P., Sheikh, A., "Buckling and Dynamic Instability Analysis of Stiffened Shell Panels," Thin-Walled Structures, Vol. 44, pp. 321-333, 2006. 
${ }^{25}$ Stevens, B., Lewis, F., Aircraft Control and Simulation, John Wiley and Sons, Hoboken, NJ, 2003.

${ }^{26}$ Adams, W., Christhilf, D., Waszak, M., Mukhopadhyay, V., Srinathkumar, S., "Design, Test, and Evaluation of Three Active Flutter Suppression Controllers," NASA TM 4338, 1992.

${ }^{27}$ Sigmund, O., "Morphology-Based Black and White Filters for topology Optimization," Structural and Multidisciplinary Optimization, Vol. 33, No. 4, pp. 401-424, 2007.

${ }^{28}$ Svanberg, K., "A Class of Globally Convergent Optimization Methods Based on Conservative Convex Separable Approximations," SIAM Journal of Optimization, Vol. 12, pp. 555-573, 2002.

${ }^{29}$ Adelman, H., Haftka, R., "Sensitivity Analysis of Discrete Structural Systems," AIAA Journal, Vol. 24, No. 5, pp. 823-832, 1986.

${ }^{30}$ Gilbert, M., "An Analytical Sensitivity Method for use in Integrated Aeroservoelastic Aircraft Design," Mechanical Systems and Signal Processing, Vol. 4, No. 3, pp. 215-231, 1990.

${ }^{31}$ Kennedy, G., "Strategies for Adaptive Optimization with Aggregation Constraints using Interior-Point Methods," Computers and Structures, Vol. 153, No. 1, pp. 217-229, 2017.

${ }^{32}$ Livne, E., "Aircraft Active Flutter Suppression: State of the Art and Technology Maturation Needs," Journal of Aircraft, Vol. 55, No. 1, pp. 410-452, 2018. 